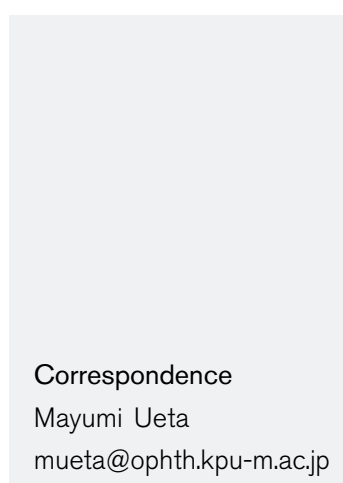

Received 2 July 2006

Accepted 14 September 2006

\section{Polyclonality of Staphylococcus epidermidis residing on the healthy ocular surface}

\author{
Mayumi Ueta, ${ }^{1}$ Tetsuya lida, ${ }^{2}$ Masako Sakamoto, ${ }^{2}$ Chie Sotozono, ${ }^{1}$ \\ Junko Takahashi, ${ }^{1}$ Kentaro Kojima, ${ }^{1}$ Kazuhisa Okada, ${ }^{2}$ Xiuhao Chen, ${ }^{2}$ \\ Shigeru Kinoshita ${ }^{1}$ and Takeshi Honda ${ }^{2}$ \\ ${ }^{1}$ Department of Ophthalmology, Kyoto Prefectural University of Medicine, Hirokoji, \\ Kawaramachi, Kamigyoku, Kyoto 602-0841, Japan \\ ${ }^{2}$ Department of Bacterial Infections, Research Institute for Microbial Diseases, Osaka \\ University, Yamadaoka, Suita, Osaka, Japan
}

\begin{abstract}
Staphylococcus epidermidis is part of the normal bacterial flora on the ocular surface. The chromosomal DNA of bacterial isolates obtained from the conjunctival sac, upper and lower lid margins, and upper and lower Meibomian glands of healthy volunteers was subjected to Smal digestion and PFGE to study the genetic diversity of the organisms. Multiple colonies were also examined of $S$. epidermidis derived from the conjunctival sac of the same subjects. Lastly, commensal bacteria were harvested from the ocular surfaces of four healthy subjects once a month for 6 months, and the genetic background of the $S$. epidermidis isolates was analysed. It was found that bacterial strains not only from different subjects but also from multiple ocular surface sites of the same subject exhibited different PFGE patterns. In five of 42 subjects multiple colonies of $S$. epidermidis were isolated from the conjunctival sac; three harboured multiple colonies with different PFGE patterns, and two manifested multiple colonies with identical PFGE patterns. $S$. epidermidis isolated from the conjunctival sac of the same subjects over a 6-month period exhibited varying PFGE patterns. The data demonstrate the polyclonality of $S$. epidermidis on the healthy ocular surface.
\end{abstract}

\section{INTRODUCTION}

In humans, the largest populations of staphylococci are usually found in areas of the skin and mucous membranes surrounding body openings. Some staphylococcus species exhibit a marked preference for certain habitats; in adults, the coagulase-positive species Staphylococcus aureus prefers the anterior nares. On the other hand, the predominant staphylococcus species Staphylococcus epidermidis is widely distributed over the body surface (Kloos \& Musselwhite, 1975).

Bacterial flora composed of S. epidermidis, Propionibacterium acnes, and other Gram-positive and -negative bacteria (Doyle et al., 1995; Hara et al., 1997), is normal in the conjunctival sac and eyelid edge, on skin, and in mucosal tissues. S. epidermidis is representative of the normal bacterial flora on the ocular surface and is most frequently isolated from the microflora of the human ocular surface.

We subjected S. epidermidis strains isolated from healthy ocular surfaces to PFGE to study the diversity in the genetic background of this bacterium. We show that $S$. epidermidis on the human ocular surface can be of polyclonal origin.

\section{METHODS}

All experimental procedures were conducted in accordance with the principles set forth in the Helsinki Declaration. The purpose of the research and the experimental protocols were explained to all participants and their prior written informed consent was obtained.

\section{S. epidermidis}

Analysis of bacterial isolates from multiple ocular surface sites of the same subject. S. epidermidis was obtained from five sites on the healthy ocular surface of volunteers, i.e. the conjunctival sac, upper and lower lid margins, and upper and lower Meibomian glands, using CultureSwab (Becton Dickinson). The isolates obtained were stored $\left(-20^{\circ} \mathrm{C}\right)$ in ANAport BIKEN culture medium (BIKEN) at the Department of Ophthalmology of Kyoto Prefectural University of Medicine; they were sent to The Research Foundation for Microbial Diseases of Osaka University on the next day.

Analysis of multiple colonies obtained from the conjunctival sac of the same subject. Commensal bacteria were obtained from the conjunctival sac of volunteers with no ocular diseases using CultureSwab at The Research Foundation for Microbial Diseases of Osaka University, and the isolates were identified without being stored in order to detect bacteria more efficiently. Multiple colonies of $S$. epidermidis derived from conjunctival sacs of the same subjects were subjected to PFGE.

Chronological analysis of bacterial changes occurring in the conjunctival sac of the same subject over a 6-month period. 
Commensal bacteria in the conjunctival sacs of four healthy volunteers were obtained once a month for 6 months using CultureSwab, and the genetic background of $S$. epidermidis was analysed by PFGE.

All isolates were identified at The Research Foundation for Microbial Diseases of Osaka University. The isolates were cultured by both direct culture and enrichment culture. In direct-culture methods, bacteria were isolated on Columbia CNA agar with $5 \%$ sheep blood (Becton Dickinson) at $37^{\circ} \mathrm{C}$ for $48 \mathrm{~h}$ aerobically, or for 5 days anaerobically. In enrichment-culture methods, isolates were cultured in thioglycolate medium (Eiken Chemical) at $37^{\circ} \mathrm{C}$ for $1-2$ weeks, and if positive for bacteria, the bacteria were isolated on Columbia CNA agar with $5 \%$ sheep blood at $37^{\circ} \mathrm{C}$ for $48 \mathrm{~h}$ aerobically or for 5 days anaerobically. $S$. epidermidis was identified using the API 20 Staph system (bioMérieux).

PFGE. For genotyping, we performed PFGE analysis of bacterial chromosomal DNA after SmaI digestion, because this is the most highly discriminating method for coagulase-negative staphylococci (Linhardt et al., 1992). The GenePath System (Bio-Rad Laboratories) was used for genetic fingerprinting. Isolates cultured overnight in brain heart infusion broth (Difco) were subjected to SmaI digestion of bacterial genomic DNA according to the manufacturer's instructions (GenePath Group I Reagent kit; Bio-Rad). PFGE patterns were obtained by separating the digested DNA on $1 \%$ agarose gels (molecular biology certified agarose, Bio-Rad) in a counter-clamped homogeneous CHEF-DR Mapper (GenePath system, Bio-Rad). The electrophoresis buffer was $0.5 \times$ TBE buffer (45 mM Tris/borate, $1 \mathrm{mM}$ EDTA); equilibration was at $14^{\circ} \mathrm{C}$ using a constant voltage of $6 \mathrm{~V} \mathrm{~cm}^{-1}$ with pulse-ramping from 5.3 to $34.9 \mathrm{~s}$ for $20 \mathrm{~h}$ at a $120^{\circ}$ angle. A lambda ladder consisting of successively larger concatemers of $48.5 \mathrm{~kb}$ DNA fragments was used as the molecular size marker. Electrophoresed gels were stained with ethidium bromide, washed with distilled water, and photographed under UV light.

DNA bands were compared by visual inspection and interpreted according to Tenover et al. (1995). Based on the number of DNA fragments that exhibited different pulse patterns, the strains were considered to be indistinguishable (identical pulse patterns), closely related (two to three fragments with different patterns), or possibly related (four to six fragments with different patterns). If more than six DNA fragments exhibited a different migration pattern, the isolates were considered to be unrelated.

\section{RESULTS AND DISCUSSION}

We first analysed commensal bacteria isolated from the conjunctival sac, upper and lower lid margins, and upper and lower Meibomian glands of healthy volunteers. $S$. epidermidis was isolated from 12 of 40 volunteers sampled at Kyoto Prefectural University of Medicine; seven of them harboured $S$. epidermidis at multiple ocular surface sites. Strains obtained from different subjects exhibited different and unrelated PFGE patterns. S. epidermidis isolated from multiple sites in a single subject did not exhibit monoclonal PFGE patterns (Fig. 1). Our results indicated that the ocular surface of healthy individuals can harbour polyclonal $S$. epidermidis.

We next harvested commensal bacteria from conjunctival sacs of 42 healthy volunteers at The Research Foundation for Microbial Diseases of Osaka University. The isolated commensal bacteria are listed in Table 1. Bacteria were isolated from the conjunctival sac of 29 of 42 volunteers (Table 1). S. epidermidis was isolated from 19 of 42 volunteers (Table 2). S. epidermidis was mainly isolated aerobically. Of these 19 subjects, five harboured multiple colonies of $S$. epidermidis, as determined by direct or enrichment culture (Table 1, volunteers 1, 2, 5, 27 and 29) and three of these five subjects harboured multiple colonies that manifested different and closely related, possibly (a)

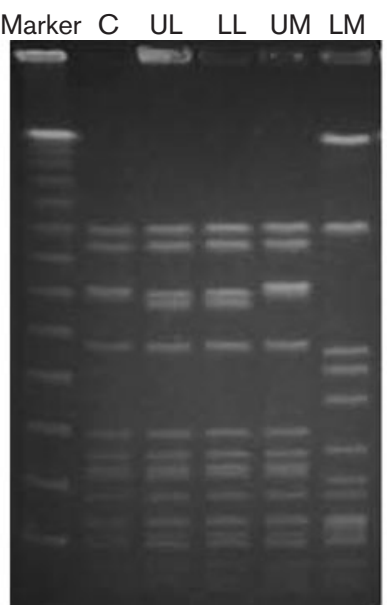

(b)

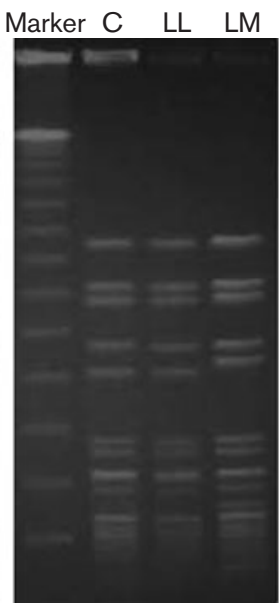

(c)

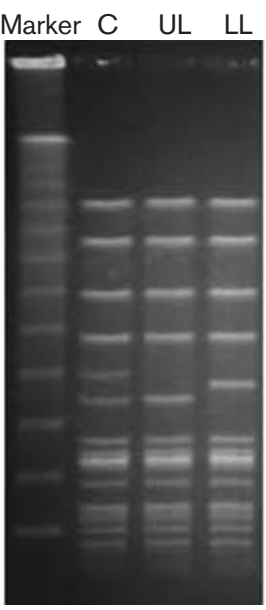

(d)

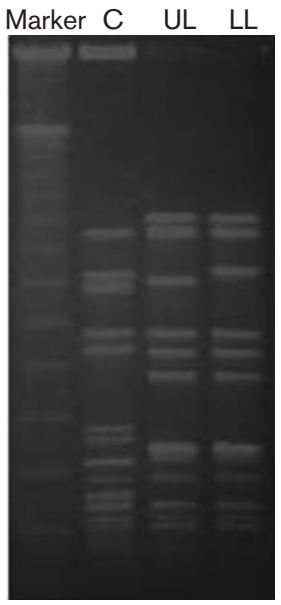

Fig. 1. S. epidermidis isolated from multiple sites on the healthy ocular surface. S. epidermidis isolated from five sites on the healthy ocular surface (conjunctival sac, upper and lower lid margins, upper and lower Meibomian glands) were analysed by PFGE (C, conjunctiva; UL, upper lid margin; LL, lower lid margin; UM, upper Meibomian gland; LM, lower Meibomian gland). The PFGE patterns of $S$. epidermidis isolated from multiple sites of the same subject were different but closely related or possibly related. Marker, molecular mass marker of concatemers of lambda phage DNA. (a-d) show S. epidermidis isolated from different subjects. 
Table 1. Commensal bacteria isolated in this study



related, or unrelated PFGE patterns (Fig. 2a, b). The other two subjects were host to multiple colonies with identical and indistinguishable PFGE patterns (Fig. 2c). These results indicate that polyclonal $S$. epidermidis may reside not only on the ocular surface, but also in the conjunctiva.

Lastly, we analysed commensal bacteria harvested once a month for 6 months from the conjunctival sac of four

Table 2. Isolation of commensal bacteria from conjunctival sacs of 42 healthy volunteers

\begin{tabular}{|lcc|}
\hline Organism & No. of isolates & As a percentage \\
\hline S. epidermidis & $19 / 42$ & $45 \%$ \\
P. acnes & $13 / 42$ & $31 \%$ \\
Others & $8 / 42$ & $19 \%$ \\
Not detected & $13 / 42$ & $31 \%$ \\
\hline
\end{tabular}

healthy volunteers (Table 3). We found that although the make-up of the microbial populations changed slightly over the course of this investigation, S. epidermidis and P. acnes remained dominant. In three out of the four subjects, we detected S. epidermidis at multiple sampling.

We analysed S. epidermidis isolated from the conjunctival sac of the same subject at different time points and found that the organisms manifested variously related or unrelated PFGE patterns. Although these patterns remained relatively similar, the S. epidermidis organisms were polyclonal and yielded multiple colonies with unrelated PFGE patterns over the course of our study. At some sampling points there was also a change in the dominant strain (Fig. 3).

To the best of our knowledge, this is the first PFGE investigation to examine the diversity in the genetic background of $S$. epidermidis residing on the healthy human ocular surface. Strains of S. epidermidis harvested 
(a)

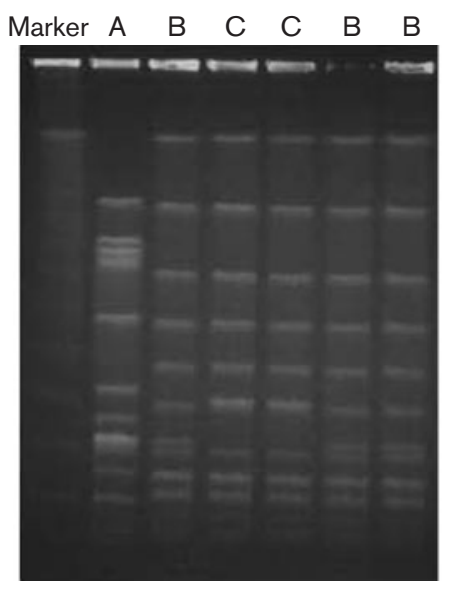

(b)

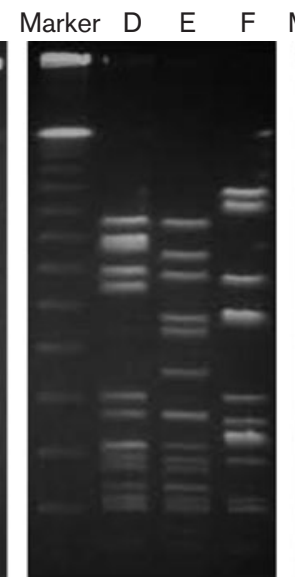

(c)

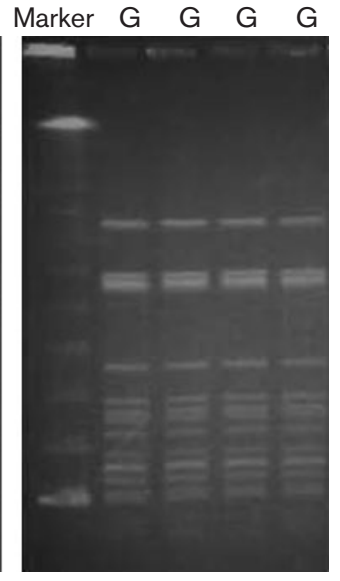

Fig. 2. Multiple colonies of $S$. epidermidis obtained from the conjunctival sac of the same subject. (a) Volunteer 1 , six of $11 \mathrm{~S}$. epidermidis colonies grown by direct culture. (A) and (B) are unrelated; (B) and (C) are closely related. (b) Volunteer 29, one colony was grown in direct culture and the other two in enriched culture. (D) and (E) are unrelated; (F) is unrelated to (D) and (E). (c) Volunteer 2, three colonies were grown in direct culture and one was grown in enriched culture. All were indistinguishable. Marker, molecular mass marker of concatemers of lambda phage DNA. from different ocular surface sites of the same donor manifested similar, albeit distinct, PFGE patterns. In five of 19 subjects with $S$. epidermidis in their conjunctival sacs, direct or enriched cultures grew multiple colonies; the PFGE patterns of each colony were similar but distinct in three, and identical in the other two. These results suggest that $S$. epidermidis inhabiting not only the ocular surface, but also the conjunctiva, are polyclonal. The harvest of S. epidermidis from the conjunctival sac of the same subject at different times revealed that while the PFGE patterns of isolates remained relatively similar over time, the organisms were polyclonal and yielded multiple colonies with unrelated PFGE patterns. A change in the dominant strain was also suggested at some sampling times. These observations confirm the polyclonality of S. epidermidis on the healthy ocular surface.

The largest populations of human staphylococci reside in areas of the skin and mucous membranes that surround openings in the body surface. Some Staphylococcus species exhibit a marked preference for certain habitats; for example, in adults, coagulase-positive $S$. aureus preferentially inhabits the anterior nares, and the predominant $S$. epidermidis species is widely distributed over the body surface (Kloos \& Musselwhite, 1975).

Bacterial flora composed of $S$. epidermidis, $P$. acnes, and other Gram-positive and Gram-negative bacteria, is normally found in the conjunctival sac or on the eyelid edge (Doyle et al., 1995; Hara et al., 1997). S. epidermidis is representative of the normal bacterial flora, and is most frequently isolated from the microflora of the human ocular surface.

Hu et al. (1995a) have reported that S. epidermidis organisms isolated from the nares of healthy subjects are polyclonal, while $S$. aureus inhabiting the nares of the same subjects is monoclonal (Hu et al., 1995b). Similarly, our results revealed that $S$. epidermidis existing as normal bacterial flora on the healthy ocular surface is polyclonal. Of 40 subjects that we

Table 3. Commensal bacteria harvested over a 6-month period from the conjunctival sac of four healthy volunteers

$\mathrm{d}$, direct culture; e, enrichment culture; the number of colonies is given after $\mathrm{d}$ or e.

\begin{tabular}{|c|c|c|c|c|}
\hline Month $^{*}$ & Volunteer 1 & Volunteer 2 & Volunteer 3 & Volunteer 4 \\
\hline 1 & Propionibacterium avidum (e1) & S. epidermidis (e1) & $\begin{array}{l}\text { S. epidermidis }(\mathrm{e} 1) \\
\text { P. acnes }(\mathrm{d} 1)\end{array}$ & $\begin{array}{l}\text { S. epidermidis (e1) } \\
\text { P. acnes }(\mathrm{e} 1)\end{array}$ \\
\hline 2 & - & $\begin{array}{l}\text { S. epidermidis }(\mathrm{e} 2) \\
\text { P. acnes }(\mathrm{d} 6)\end{array}$ & $\begin{array}{l}\text { S. epidermidis }(\mathrm{d} 3, \mathrm{e} 1) \\
\text { P. acnes }(\mathrm{d} 4) \\
\text { P. granulosum }(\mathrm{d} 1)\end{array}$ & S. epidermidis (e1) \\
\hline 3 & P. acnes $(\mathrm{e} 1)$ & S. epidermidis (d1, e2) & S. epidermidis (e2) & $\begin{array}{l}\text { S. epidermidis }(\mathrm{d} 1) \\
\text { P. acnes }(\mathrm{e} 1)\end{array}$ \\
\hline 4 & - & S. epidermidis (e1) & S. epidermidis (e1) & - \\
\hline 5 & P. acnes $(\mathrm{e} 1)$ & $\begin{array}{l}\text { S. epidermidis }(\mathrm{e} 1) \\
\text { P. acnes }(\mathrm{d} 5)\end{array}$ & $\begin{array}{l}\text { P. acnes }(\mathrm{d} 21) \\
\text { S. aureus }(\mathrm{e} 1)\end{array}$ & - \\
\hline 6 & S. epidermidis (e1) & S. hominis (e1) & S. epidermidis (e1) & - \\
\hline
\end{tabular}

${ }^{\star}$ The month in which the samples were collected. 
(a)

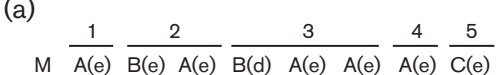

(b)
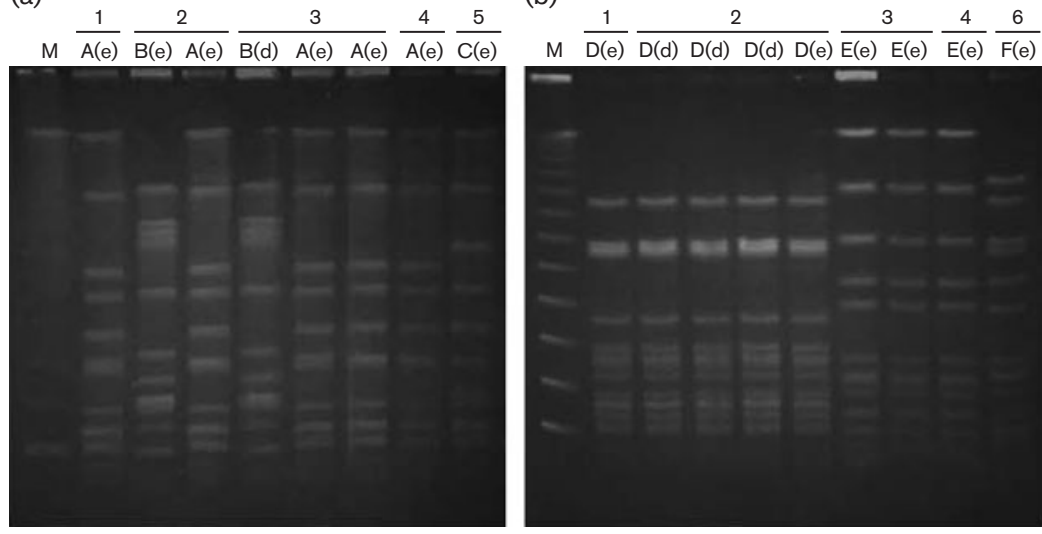

Fig. 3. $S$. epidermidis harvested at different times from the conjunctival sac of the same subjects. (a) A 42-year-old woman. Of eight colonies, one was grown in direct and seven in enriched culture. At four sampling times (months 1, 2, 3 and 4), the same clone (A) of $S$. epidermidis was detected. At 2- and 3-month sampling, two clones (A and $B$ ) of $S$. epidermidis were detected. Sampling on the fifth month detected one clone (C) of $S$. epidermidis; it was closely related to (A). (b) A 36-year-old man. Of nine colonies, three were grown in direct and six in enriched culture. At 1- and 2-month sampling, the same clone (D) of $S$. epidermidis was detected. At 3- and 4-month sampling, clone (E) was detected. It was unrelated to (D). In the sixth month, clone $(F)$, unrelated to $(E)$, was detected. $\mathrm{M}$, molecular mass marker of concatemers of lambda phage DNA. examined, two harboured S. aureus at multiple ocular sites. The PFGE patterns of $S$. aureus isolated from multiple ocular sites of a single subject were identical and indistinguishable (data not shown), supporting the hypothesis that $S$. aureus organisms residing in the human nares and ocular surface are monoclonal. In contrast to S. epidermidis, which is present in the nares of almost all healthy individuals, $S$. aureus is found in the nares of only about $30 \%$ of healthy subjects (Hu et al., 1995a). According to Doyle et al. (1995), only $6 \%$ of healthy subjects harbour $S$. aureus on the ocular surface, while $90 \%$ host S. epidermidis. As S. aureus is an ophthalmic pathogen that can induce bacterial conjunctivitis, keratitis and blepharitis (Kupferman \& Leibowitz, 1977; McCulley et al., 1982; Prentice et al., 1977), it may harbour pathogenicity even when present in the nares of healthy individuals.

Due to its ubiquity and relatively low virulence, $S$. epidermidis has been considered as a clinically insignificant contaminant when isolated from clinical samples (Eng et al., 1982). However, S. epidermidis is increasingly recognized as an important agent of nosocomial infection (Kloos \& Bannerman, 1994; Pfaller \& Herwaldt, 1988), and many of these infections have been traced to monoclonal $S$. epidermidis (Burnie et al., 1997; Murono et al., 2003; Nouwen et al., 1998). In the majority of immunocompromised patients, the causative organisms of bloodstream infections originate primarily from the patients' own flora (Kloos \& Bannerman, 1994; Murono et al., 2003).

Although S. epidermidis is a common component of the normal ocular flora, it can lead to chronic blepharitis, conjunctivitis and keratitis, especially in immunocompromised hosts (Baum, 1978; Pinna et al., 1999), suggesting that opportunistic infection with $S$. epidermidis is reflective of the status of the host. We encountered one instance in which monoclonal S. epidermidis was isolated from multiple sites in both eyes (data not shown). The host was an immunocompromised patient who had undergone bone marrow transplantation and developed superficial punctuate keratitis (SPK). Disruption of the balance between $S$. epidermidis, which is a commensal bacterium on the ocular surface, and the status of this host, may have resulted in the monoclonality of the bacteria. We postulate that a good balance between commensal bacteria and hosts maintains the polyclonality of $S$. epidermidis and that a weakened host status may contribute to its pathogenicity. Studies are under way in our laboratories to examine this issue.

In summary, we examined the diverse genetic background of S. epidermidis isolated from the ocular surface of healthy subjects, and found that the bacterium may be polyclonal on healthy ocular surfaces.

\section{REFERENCES}

Baum, J. L. (1978). Current concepts in ophthalmology. Ocular infections. N Engl J Med 299, 28-31.

Burnie, J. P., Naderi-Nasab, M. K., Loudon, W. \& Matthews, R. C. (1997). An epidemiological study of blood culture isolates of coagulase-negative staphylococci demonstrating hospital-acquired infection. J Clin Microbiol 35, 1746-1750.

Doyle, A., Beigi, B., Early, A., Blake, A., Eustace, P. \& Hone, R. (1995). Adherence of bacteria to intraocular lenses: a prospective study. Br J Ophthalmol 79, 347-349.

Eng, R. H., Wang, C., Person, A., Kiehn, T. E. \& Armstrong, D. (1982). Species identification of coagulase-negative staphylococcal isolates from blood cultures. J Clin Microbiol 15, 439-442.

Hara, J., Yasuda, F. \& Higashitsutsumi, M. (1997). Preoperative disinfection of the conjunctival sac in cataract surgery. Ophthalmologica 211 (Suppl. 1), 62-67.

Hu, L., Umeda, A. \& Amako, K. (1995a). Typing of Staphylococcus epidermidis colonizing in human nares by pulsed-field gel electrophoresis. Microbiol Immunol 39, 315-319. 
Hu, L., Umeda, A., Kondo, S. \& Amako, K. (1995b). Typing of Staphylococcus aureus colonising human nasal carriers by pulsed-field gel electrophoresis. J Med Microbiol 42, 127-132.

Kloos, W. E. \& Bannerman, T. L. (1994). Update on clinical significance of coagulase-negative staphylococci. Clin Microbiol Rev 7, 117-140.

Kloos, W. E. \& Musselwhite, M. S. (1975). Distribution and persistence of Staphylococcus and Micrococcus species and other aerobic bacteria on human skin. Appl Microbiol 30, 381-385.

Kupferman, A. \& Leibowitz, H. M. (1977). Topical antibiotic therapy of staphylococcal keratitis. Arch Ophthalmol 95, 1634-1637.

Linhardt, F., Ziebuhr, W., Meyer, P., Witte, W. \& Hacker, J. (1992). Pulsed-field gel electrophoresis of genomic restriction fragments as a tool for the epidemiological analysis of Staphylococcus aureus and coagulase-negative staphylococci. FEMS Microbiol Lett 74, 181-185.

McCulley, J. P., Dougherty, J. M. \& Deneau, D. G. (1982). Classification of chronic blepharitis. Ophthalmology 89, 1173-1180.

Murono, K., Hirano, Y., Koyano, S., Ito, K. \& Fujieda, K. (2003). Molecular comparison of bacterial isolates from blood with strains colonizing pharynx and intestine in immunocompromised patients with sepsis. J Med Microbiol 52, 527-530.
Nouwen, J. L., van Belkum, A., de Marie, S., Sluijs, J., Wielenga, J. J., Kluytmans, J. A. \& Verbrugh, H. A. (1998). Clonal expansion of Staphylococcus epidermidis strains causing Hickman catheter-related infections in a hemato-oncologic department. J Clin Microbiol 36, 2696-2702.

Pfaller, M. A. \& Herwaldt, L. A. (1988). Laboratory, clinical, and epidemiological aspects of coagulase-negative staphylococci. Clin Microbiol Rev 1, 281-299.

Pinna, A., Zanetti, S., Sotgiu, M., Sechi, L. A., Fadda, G. \& Carta, F. (1999). Identification and antibiotic susceptibility of coagulase negative staphylococci isolated in corneal/external infections. $\mathrm{Br}$ J Ophthalmol 83, 771-773.

Prentice, M. J., Hutchinson, G. R. \& Taylor-Robinsin, D. (1977). A microbiological study of neonatal conjunctivae and conjunctivitis. $\mathrm{Br}$ J Ophthalmol 61, 601-607.

Tenover, F. C., Arbeit, R. D., Goering, R. V., Mickelsen, P. A., Murray, B. E., Persing, D. H. \& Swaminathan, B. (1995). Interpreting chromosomal DNA restriction patterns produced by pulsed-field gel electrophoresis: criteria for bacterial strain typing. J Clin Microbiol 33, 2233-2239. 